\title{
Angustia en cuidadores de niños con fiebre: análisis del concepto. Modelo híbrido ${ }^{\dagger}$
}

\author{
Anguish in caregivers of children with fever: analysis of the concept. Hybrid model \\ Angustia em cuidadores de crianças com febre: análise do conceito. Modelo híbrido \\ Ana Ligia Escobar-Tobón* \\ María Mercedes Arias-Valencia ** \\ Ángela María Salazar-Maya ${ }^{* * *}$
}

\section{Resumen}

Objetivo: Analizar el concepto de angustia en cuidadores informales de niños con fiebre. Materiales y Método: Se seleccionó el modelo híbrido de análisis de concepto que consiste en tres fases: la teórica en la que se realizó una amplia revisión de la literatura; luego la de trabajo de campo, que consistió en la recopilación y análisis de datos cualitativos, a través de 15 entrevistas a cuidadores informales; y, por último, la fase analítica en la que se analizaron, compararon y consolidaron los datos de la fases teórica y de trabajo de campo, para producir una definición más refinada del concepto. Resultado: La angustia en el cuidador informal se define como: un estado afectivo transitorio que surge en el cuidador que vive una condición de incertidumbre sobre algo desconocido que subyace al proceso febril, a sus manifestaciones, al acompañamiento y a las consecuencias del signo o síntoma de la fiebre, provocando una sensación de zozobra. Conclusiones: El análisis del concepto de angustia en los cuidadores informales, permitió distinguirlo de conceptos relacionados e identificar situaciones que pueden afectar la salud física y mental del cuidador cuando ésta se prolonga; además posibilita a la disciplina de la enfermería generar aportes sobre la práctica del cuidado, especialmente en lo que tiene que ver con la incertidumbre en el cuidador relacionada con la presencia de fiebre en el niño.

Palabras clave: Enfermería, cuidadores, estrés psicológico, fiebre, investigación cualitativa.

\section{Abstract}

Objective: Analyze the concept of anguish on informal caregivers of children with fever. Materials and Method: The hybrid model of analysis of concept was selected

${ }^{\dagger}$ Artículo derivado de la investigación: Significados que construyen los cuidadores informales y el personal de salud, sobre el cuidado al niño con fiebre.

\section{Autor de correspondencia}

* $\triangle$ Enfermera. Magister en Educación y Desarrollo Humano, candidata a Doctora en Enfermería. Docente Universidad de Antioquia. Colombia. Correo: ana.escobar@udea.edu. co. (iD https://orcid.org/0000-0003-02229522. Medellín, Colombia.

** Enfermera, Doctora en Salud Pública ENSP-FIOCRUZ, Brasil. Docente Universidad de Antioquia. Correo: mercedes.arias@udea. edu.co (iD https://orcid.org/0000-0003-38765976. Medellín, Colombia.

${ }^{* * *}$ Enfermera, Doctora en enfermería, Docente Universidad de Antioquia. Correo: angela.salazar@udea.edu.co (D) https://orcid. org/0000-0001-7599-1193. Medellín, Colombia.
Este es un artículo bajo la licencia CC BY (https://creativecommons.org/ licenses/by/4.0/) @) (i) 
ISSN-PRINT

1794-9831

E-ISSN 2322-7028

Vol. 15 No. 2

Jul - Dic 2018

Cúcuta, Colombia

and consists of 3 phases: the theoretical stage where an ample review of literature was performed; the fieldwork stage, which consisted on the recollection and analysis of quantitative data through 15 interviews to informal caregivers; and lastly the analytic phase, where the data from the theoretical and the fieldwork stages were analyzed, compared, and consolidated to produce a more refined definition of the concept. Result: The anguish of the caregiver is defined as: an affective transitory state that rises in the caregiver who experiences a condition of uncertainty about something unknown, underlying in the fever process, its manifestations, the accompaniment, and the consequences of the signs or symptoms of fever, generating a feeling of anxiety. Conclusions: The analysis of the anguish concept on informal caregivers, allowed distinguishing it from different related concepts and also identify situations that can affect the physical and mental health of the caregiver when the situation prolongs, also enabling the nursing discipline to generate contributions about the care practice, especially with the uncertainty caused by the disease (fever) of the child on the caregiver.

Keywords: Nursing, caregivers, psychological stress, fever, qualitative research.

\section{Resumo}

Objetivo: Analisar o conceito de angustia em cuidadores informais de crianças com febre. Materiais e Método: Selecionou-se o modelo híbrido da análise de conceito que consiste em três fases: uma teórica na qual se realizou uma ampla revisão da literatura; depois uma de trabalho de campo, que consistiu na recopilação e análise de dados qualitativos através de 15 entrevistas a cuidadores informais; e, por último, a fase analítica na qual se analisaram, compararam e consolidaram os dados das fases teórica e de trabalho de campo, para produzir uma definição mais aperfeiçoada do conceito. Resultado: A angustia no cuidador informal se define como: um estado afetivo transitório que surge no cuidador que vive uma condição de incerteza sobre algo desconhecido que subjaz ao processo febril, a suas manifestações, ao acompanhamento e às consequências do sinal ou sintoma da febre, provocando uma sensação de ansiedade. Conclusões: A análise do conceito de angustia nos cuidadores informais, permitiu distingui-lo de conceitos relacionados e identificar situações que podem afetar a saúde física e mental do cuidador quando esta é prolongada; além disso, possibilita à disciplina da enfermagem gerar contribuições sobre a prática do cuidado, especialmente no que tem que ver com a incerteza relacionada com a doença no cuidador.

Palavras-chave: Cuidadores, enfermagem, estresse psicológico, febre, pesquisa qualitativa.

\section{Introducción}

La angustia se ha abordado desde la filosofía, la psicología y la psiquiatría, las cuales dan cuenta de ella como un estado emocional que se produce al percibir una amenaza futura $(1,2)$. Múltiples investigaciones muestran el impacto en la salud mental y física de los cuidadores al asumir el cuidado de seres queridos; entre las afecciones mentales encontradas están: el miedo, problemas nerviosos, irritabilidad, soledad, desesperanza, tristeza, ansiedad, disgusto, hostilidad, culpa, sentimientos de indefensión, depresión, hasta pensamientos de abandono y de suicidio (3). En lo físico, problemas osteomusculares, cefalea, astenia, malestar general, trastornos del sueño, hipertensión, entre otros (4).

Existen estudios que muestran una clara relación entre la angustia psicológica de los pacientes y la angustia de los cuidadores, y la influencia en la calidad de vida de estos (5). Al respecto, los cuidadores como principales proveedores de cuidado del paciente, asumen altas demandas físicas y emocionales que pueden generar en grados significativos de angustia que repercuten en su calidad de vida, en su ámbito familiar, personal, laboral, y, de esta manera, pueden afectar su entorno al situarlos en una situación de alta vulnerabilidad, estrés y "carga", definida como: "aquel agotamiento mental y ansiedad frente al cuidado, el cual además está relacionado con afectaciones en la salud física" (6).

De igual manera, se encuentran estudios que muestran el impacto en los cuidados, lo que evidencia que entre un tercio y la mitad de los cuidadores sufren trastornos psicológicos significativos y experimentan mayor riesgo de sufrir problemas físicos, emocionales y sociofamiliares frente a la población general; así mismo, pueden sufrir altas dosis de estrés que influyen negativamente en el equilibrio y la integridad 
de la familia. En ese sentido, el rol de los cuidadores se torna difícil por la complejidad de tareas que asumen y que por tanto los ubica en una posición de vulnerabilidad, estrés y carga (7).

Sin embargo, aunque se encuentran numerosas investigaciones relacionadas con la angustia en los cuidadores de niños (8), éstas se han centrado en niños con enfermedades crónicas (9) y/o mortales (10); poco se conoce sobre la naturaleza de la angustia en los cuidadores de niños con enfermedades agudas como la fiebre, que tiene la característica de ser transitoria. Si bien la enfermedades agudas son consideradas de corta duración (11), ello no significa que no conlleven implicaciones emocionales importantes para el cuidador que repercutan en él y en el sujeto de cuidado. Por ello es necesario discernir sobre el concepto de angustia en los cuidadores, si se tiene en cuenta que la falta de aclaración y de visibilidad del mismo, afecta las prácticas del cuidado. El aporte del trabajo de campo de ésta investigación, hará referencia a la angustia en cuidadores de niños en estados nosológicos agudos.

Analizar el concepto de angustia en los cuidadores informales de niños con fiebre, es relevante para la enfermería como lo indican Zarit et al. (12), para quienes el análisis de un concepto es útil para comprenderlo y refinarlo además de ser utilizado en una variedad de configuraciones y de maneras. Al reducir la ambigüedad asociada a un concepto, este se puede incluir de manera significativa en el desarrollo de las taxonomías de enfermería

El propósito de analizar este concepto, es aclarar su base conceptual en la práctica de la enfermería y así determinar el estado de conocimiento y presentar una estimación actual para su uso probable.

De esta manera, se convierte en un desafío para la enfermería identificar cómo la angustia puede impactar en la salud de los cuidadores informales de niños con fiebre y/o con enfermedades agudas; asimismo, permitiría implicar la disciplina en el diseño de estrategias de intervención para este grupo.

Por lo anterior este artículo pretende, a la luz de lo que ha significado y cómo hoy se resignifica la angustia en los cuidadores informales, analizar el concepto, de manera que facilite su uso en la enfermería y estimule respecto a la reflexión y la acción del profesional como cuidador. Esto supone el desarrollo de una definición que describa sus atributos a partir de un proyecto específico de investigación, teniendo en cuenta sus implicaciones en la práctica de la enfermeria.

\section{Objetivos}

\section{Objetivo General}

Analizar el concepto de angustia en cuidadores informales de niños con fiebre.

\section{Objetivos específicos}

- Identificar los antecedentes de la angustia del cuidador informal del niño con fiebre.

- Describir los atributos de la angustia del cuidador del niño con fiebre.

- Comprender las consecuencias de la angustia de los cuidadores de niños con fiebre.

\section{Materiales y Métodos}

El análisis de concepto es una técnica que ayuda a usar el pensamiento y la comunicación para dilucidarlos en sus múltiples aplicaciones y significados. Así, se consideró examinar el concepto de angustia en los cuidadores informales de niños con fiebre, el cual se analizó con el método híbrido (13) que permite el análisis teórico, la investigación empírica y la inclusión de personas, lo cual enriquece la revisión sistemática de la literatura en la investigación. En éste proyecto se contemplan las tres fases del modelo.

La fase de trabajo de campo se inició con las entrevistas a los cuidadores informales acerca del cuidado del niño con fiebre. El escenario fue el servicio de pediatría de un hospital de segundo nivel de complejidad, ubicado en el Oriente de Antioquia, departamento de Colombia. Los participantes seleccionados fueron aquellos cuidadores informales que hubieran tenido la experiencia de cuidar a un niño con fiebre. El acercamiento a ellos se realizó cara a cara, se les informó sobre la investigación, previo aval institucional. Ningún cuidador se negó a participar. Las preguntas que llevaron a indagar y profundizar en la angustia como sentimiento comúnmente expresado por los cuidadores, fueron: ¿qué siente usted cuando cuida al niño que tiene fiebre? ¿Podría describir más este sentimiento?. Con estos interrogantes se buscó detallar lo que los cuidadores definían como angustia 
ISSN-PRINT

1794-9831

E-ISSN 2322-7028

Vol. 15 No. 2

Jul - Dic 2018

Cúcuta, Colombia y los sentimientos que estuvieron inmersos. Se realizaron 13 entrevistas semiestructuradas con una duración aproximada de 45 minutos y 2 entrevistas en profundidad. Se destaca que las 15 participantes fueron madres.

Las entrevistas se efectuaron después de brindar información y ser firmado el consentimiento informado, en lugares escogidos por las cuidadoras. Estas fueron grabadas $y$ transcritas por una investigadora. Las siglas con las que se identifican los participantes en este artículo son: C: Cuidador informal seguido del número que indica el orden de la entrevista. La recolección de información se llevó a cabo de marzo de 2015 a septiembre de 2016. Se realizaron notas de: campo, teóricas y metodológicas que sirvieron de apoyo en el procesamiento y el análisis del concepto.

En esta etapa surgió la necesidad de profundizar y clarificar el concepto de angustia en el cuidador, debido a que emergió de manera significativa en el análisis, mediante definiciones complejas, delimitaciones conceptuales y clasificaciones ambiguas al revisar la literatura.

Luego, la búsqueda de la literatura dio inicio a la fase teórica, para tratar de capturar la esencia general del concepto y cómo este se define, se utiliza y se mide desde diferentes posturas disciplinares como la psicología, la psiquiatría y la enfermería.

Las definiciones sobre concepto se indagaron en una variedad de fuentes, incluyendo la literatura publicada en las bases de datos web of Science, Scopus, Science Direct, PubMed, Ebsco, de los últimos 6 años (2011 -2017), además de libros y diccionarios online. También, se utilizaron los siguientes términos de búsqueda en el idioma inglés, español y portugues: "distress" AND "caregivers"AND "children", "quality of life" AND "distress" AND "caregivers"AND "children", "caregivers" AND "distress phisicological"AND "children". Como resultado se encontraron 220 artículos de los cuales, después de eliminar los duplicados y revisar el resumen quedaron 173 artículos. Posteriormente, se eliminaron 148, conformados de 60 que no tenían el texto completo y 88 por enfatizar en la angustia del paciente y no del cuidador. Fue así como se seleccionaron 25 artículos que cumplían con los siguientes criterios de inclusión: que fuesen arbitrados y que hicieran referencia a los sucesos o sentimientos que anteceden a la angustia y/o a las definiciones y/o a las características y efectos de la misma en los cuidadores (ver Figura 1).

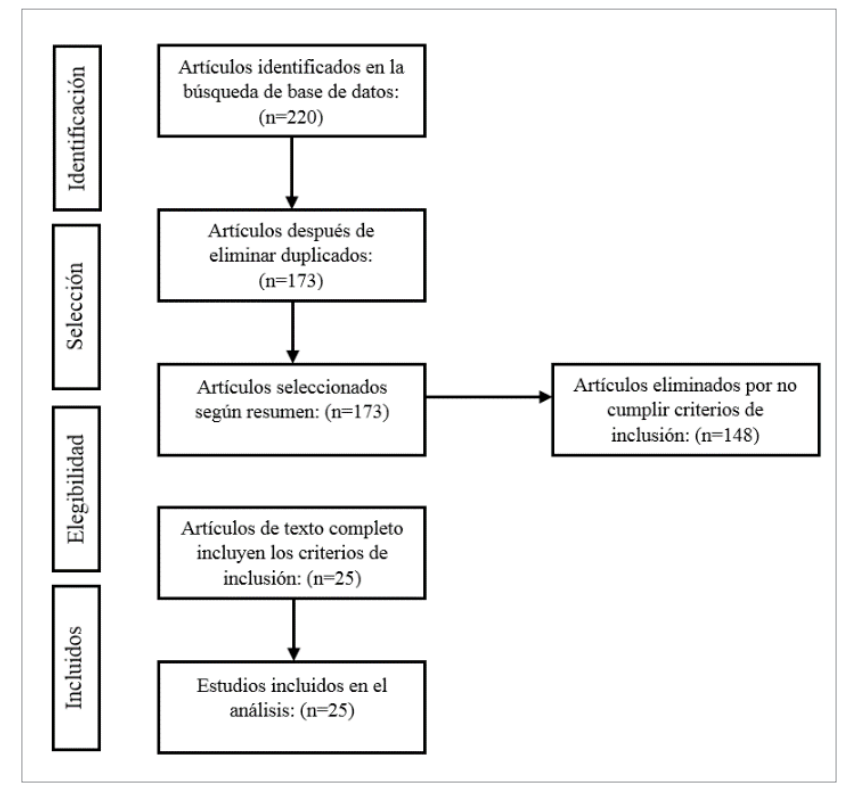

Figura1.Diagramadeflujodelasdecisionestomadasparala selección, evaluación crítica y la extracción de datos para fase teórica.

Fuente: Autor. 
Después de leer y analizar estos artículos en profundidad, se procedió a consignar la síntesis en una planilla diseñada previamente, la cual contenía: nombre del artículo, autores, atributos, diferencias y similitudes con otros conceptos, antecedentes, consecuencias y otros resultados que aportaron al esclarecimiento del concepto.

Por último, en la fase analítica, los datos de la fase de trabajo de campo se compararon y analizaron con los hallazgos encontrados en la fase teórica, para presentar una definición que aportara nuevas visiones al concepto de angustia, con respaldo y soporte de la reflexión teórica y de las perspectivas de los participantes.

El proyecto fue avalado por el comité de ética de la Facultad de Enfermería de la Universidad de Antioquia (Acta CEI-FE 03 de febrero 27 de 2015).

\section{Resultados}

\section{Fase teórica}

La definición de angustia, según el diccionario de Oxford (14), es dolor severo, sufrimiento mental o infelicidad. Se deriva del latín angustus, que quiere decir "estrecho". En la actualidad, angustia se utiliza como sinónimo de ansiedad, al considerarlos como estados psicológicos displacenteros acompañados, de manera frecuente, de síntomas fisiológicos, describiéndose como expectación penosa o desasosiego ante un peligro impreciso.

Para el Diccionario de la lengua española (15), la angustia proviene del latín angustus, "angostura", "dificultad". La define como: "1. Aflicción, congoja, ansiedad. 2. Temor opresivo sin causa precisa. 3. Aprieto, situación apurada. 4. Sofoco, sensación de opresión en la región torácica o abdominal. 5. Dolor o sufrimiento. 6. Náuseas (gana de vomitar). 7. Estrechez del lugar o del tiempo.

El padre del psicoanálisis, Sigmund Freud, quien dedicó mucho tiempo a su estudio, clasifica y diferencia la angustia entre patológica y no patológica, esta última la define como: ...la angustia realista, aquella que surge de manera real y comprensible y que se da como una reacción frente a la percepción de un peligro exterior, es decir de un daño esperado, unida al reflejo de la huida y en la que se percibe una manifestación de auto conservación. Las oportunidades en que se presente la angustia (es decir, frente a qué objetos y en qué situaciones) dependerán en buena parte, como es natural, del estado de nuestro saber y de nuestro sentimiento de poder, respecto del mundo exterior (2).

La angustia emocional se considera como una respuesta afectiva a una pérdida real o percibida, que provoca una amenaza a una persona o puede frustrar sus. Está compuesta por múltiples estados emocionales, incluidas la ira, la ansiedad, la depresión, el miedo (físico) y la impotencia (16).

La angustia se define como la emoción más experimentada por todos los seres humanos. Dentro de las características principales y diferenciales con la ansiedad están el efecto de inmovilización y el llevar al sobrecogimiento en innumerables ocasiones y al desespero. Es como una emoción compleja, percibida como desagradable, la cual tiene efectos tanto psíquicos como orgánicos. Ésta además aparece cuando el ser humano se siente amenazado por algo (17).

Otra característica importante es la pérdida de la capacidad de actuar de manera voluntaria y libre, lo que quiere decir que hay pérdida de la capacidad de dirigir los actos, hay un sentimiento de impotencia. La reacción ante la angustia es de paralización, con predominio de los síntomas físicos; además, el grado de nitidez de captación del fenómeno se encuentra atenuado (2).

Conceptos relacionados. Aunque en la actualidad el concepto de angustia se ha utilizado indistintamente como sinónimo de ansiedad, debido a los efectos desagradables tanto psicológico como fisiológico, lo que se pretende con la clarificación de estas nociones es hacer las distinciones entre ambas, de manera que permitan hacer diferenciable y fácilmente identificable el concepto de angustia.

La ansiedad alude a un estado de agitación e inquietud desagradable caracterizado por la anticipación del peligro y en el cual el fenómeno se percibe con total nitidez; mientras que en la angustia el fenómeno no es claro (2).

Después de haber analizado el concepto de angustia encontrado en la literatura, así como los conceptos relacionados, se presentan las tablas en las que se incluyen las referencias de los 25 artículos revisados que contienen los antecedentes, atributos y consecuencias de la angustia en el cuidador. 
ISSN-PRINT

1794-9831

E-ISSN 2322-7028

Vol. 15 No. 2

Jul - Dic 2018

Cúcuta, Colombia
Los Antecedentes (12) permiten ampliar la mirada y ubicar el contexto en el cual se utiliza este concepto en los cuidadores, para identificar lo que propicia su aparición. En la tabla 1, se presentan los números de las referencias de los artículos que contienen los antecedentes de la angustia del cuidador revisados en la literatura.

Tabla 1. Referencias artículos que contienen los antecedentes de la angustia del cuidador

\begin{tabular}{ll}
\hline \multirow{2}{*}{ Angustia del cuidador } & \multicolumn{1}{c}{ Antecedentes } \\
\cline { 2 - 3 } & \multicolumn{1}{c}{ Carga del cuidado $(5,7,16,18-21)$} \\
Madres $(7,20,22,23)$ \\
Falta apoyo $(18,22-24)$ \\
Problemas cognitivos, deterioro funcional y/o \\
enfermedades graves del sujeto de cuidado $(5,20,21,25)$ \\
Incertidumbre sobre la enfermedad $(5,20,23,24)$ \\
Corresidencia $(20,23,26)$ \\
\hline
\end{tabular}

Fuente: Autores.

Los atributos constituyen una definición más real, debido a que va más allá de la establecida en un diccionario, trata de identificar lo que subyace en él, lo que lo representa y es característico de su uso. La tabla 2 muestra las referencias de los artículos que contienen los atributos de la angustia.

Tabla 2. Referencias de artículos que contienen los atributos de la angustia del cuidador

\begin{tabular}{l|l}
\hline \multirow{2}{*}{ Angustia del cuidador } & \multicolumn{1}{c}{ Atributos } \\
\cline { 2 - 3 } & \multicolumn{1}{c}{ Horarios/actividades interrumpidas $(18,25)$} \\
& Insomnio-vigilia $(21,23)$ \\
& Fatiga $(8,25)$ \\
& Miedo $(8,21,23,27)$ \\
& Ansiedad $(5,8,18,28)$ \\
& Tensión $(25)$ \\
& Preocupación $(5,18,20,22,25,28,29)$ \\
& Estrés $(5,7,8,20,23-25)$ \\
& Impotencia-disminución de la percepción de autoeficacia \\
\hline & $(7,8,20,21,25,26,28,29)$ \\
\hline
\end{tabular}

Fuente: Autor

Las consecuencias son aquellos eventos, situaciones, incidentes y fenómenos que pueden ocurrir como resultado de la aparición de este estado, cuyo concepto a su vez, puede estimular nuevas ideas o vías de investigación relacionadas con otros conceptos. En la tabla 3, se muestran las consecuencias de la angustia del cuidador identificadas en la literatura revisada. 
Tabla 3. Referencias artículos que contienen las consecuencias de la angustia del cuidador

Bienestar físico reducido $(7,23)$

Bienestar emocional reducido $(5,8)$

Tristeza (26)

Repercusión en la calidad de vida $(5,16)$

Depresión $(5,7,18,21,22,24,29,30)$

Irritabilidad $(8,24,29)$

Perturbación ambiente familiar $(5,7,21,29-31)$

Interrupción vida social $(5,8,18,21)$

Limitación del tiempo libre $(21,25)$

Dificultades laborales $(8,16,21)$

Problemas económicos $(5,7,16,20,22,26,28)$

Recursos insuficientes para el cuidado $(8,22)$

Fuente: Autor.

\section{Fase de trabajo de campo}

Los datos obtenidos arrojaron resultados sobre los antecedentes, atributos y consecuencias, además de algunas características de los cuidadores que presentan angustia. Los antecedentes de la angustia se relacionan con: ver al niño diferente, la incertidumbre y la posibilidad de que tengan convulsiones. La fuente principal de angustia la constituyen los cambios que se observan, tales como:

"Estar decaído, estar agitado, no querer comer y estar como muy lloroncito" (C8). "Con mi hijo yo me angustio mucho porque usualmente $\mathrm{N}$ a veces es como muy lloroncito... y cuando tiene fiebre es una cosa terrible... llora demasiado y se ve muy agitadito..." (C8). Los motivos de angustia comienzan cuando el niño se percibe "diferente", es decir, se ve de forma inusual, contrario a lo de siempre, lo normal..." (C8).

Lo "usual" también se refiere no solo a lo que se observa sino también a lo que se hace:

"Cuando por ahí a los ocho días empezó con fiebre y uyyyy para mí él empieza con fiebre y normal... lo empecé a manejar con acetaminofén... al otro día amaneció más indispuesto, pero nada respiratorio sino como la fiebre... Yo estando en mi casa lo veo como aburridito, adinámico, yo digo: está haciendo fiebre... Ahí sí me empecé a angustiar... porque ya ni con el $\mathrm{N}$ (nombre de medicamento comercial para disminuir la fiebre) que es lo que le damos, que es el único que le ha servido a él con las temperaturas, cuando no, $38,5^{\circ} \mathrm{C}$, $39^{\circ} \mathrm{C}$... ni con el baño... le daba el medicamento, esperaba un poquito, lo bañaba... y nada..." (C4).
Respecto a los procesos nosológicos agudos, que tiene la fiebre como síntoma, los cuidadores expresan:

"Lo primero que yo siento cuando el niño tiene fiebre es angustia... porque uno no sabe nada... pues uno al verlos a ellos enfermos, uno sufre... ¡Ay! qué tendrá... qué le irá a dar... uno se angustia..." (C2). En estos procesos, la incertidumbre es angustiosa, tal como se relata: "yo no sé..., no, no sé... no sé... como que me da susto... yo pienso que... tantas cosas... (C2). Y también expresa:

"Qué será, qué serán esas fiebres, uno como que empieza a inquietarse ...cómo a preocuparse.... entonces uno empieza como a angustiarse ....qué será...o será que es normal en ellos...es que es la única niña que yo tengo.. uno empieza como mamá primeriza a experimentar..." (C10).

Y la tercera fuente y que causa gran motivo de angustia es la posibilidad que presenten convulsiones:

“...por convulsionar... no es que llegue allá y convulsione, es que de pronto... de pronto... Con $38^{\circ} \mathrm{C}, 38,5^{\circ} \mathrm{C}$, sí genera pues un poquito de angustia, pero no tanto que llegue a $39^{\circ} \mathrm{C}, 39,5^{\circ} \mathrm{C} \ldots$ ahí sí da una angustia que es más grande, porque de pronto puede convulsionar..." (C4).

Atributos de la angustia: en los datos se encontraron atributos de la angustia que aluden especialmente a intranquilidad, susto, preocupación, zozobra, miedo, impotencia, parálisis, confusión y desespero. La impotencia la expresan los cuidadores como no poder hacer nada (C8); la parálisis la refieren en 
términos de "no ser capaz "(C11); la confusión y el desespero como "no saber manejar" (C15), en este caso, se refieren a la situación o el proceso febril. De esta manera con la expresión "no saber manejar" están indicando que carecen del conocimiento y la experiencia que requieren para afrontar la situación de cuidado.

ambién los cuidadores aludieron al susto, al miedo y a la intranquilidad como sinónimos de angustiosa y se refleja: "En las noches usted le da el jarabe y se acuesta... usted lo toca a raticos... y está hirviendo... uno es intranquilo... sí, porque generalmente están indispuestos... se ven indispuestos" (C8); "Eh...Si... no...Si, le estuve tomando temperatura y no le subió... era por ahí $36.5^{\circ} \mathrm{C}$...pues no le subió mucho no...la otra si era $37.8^{\circ} \mathrm{C} \ldots$ ya casi llegar a $38^{\circ} \mathrm{C}$ y ya eso si me da como susto...cuando va llegando a $38^{\circ} \mathrm{C}$.. Ya si esto va como en serio..." (C12). En cuanto al miedo: "Sí, eso me ha pasado... y como le digo... cuando yo veo que pasa eso... y vuelvo y... le digo a mí me da miedo... darle el acetaminofén antes de tiempo... a mí me da miedo" (C2).

Además se encontraron señales de impotencia y parálisis:

“...al ver que pasan las horas y el niño sigue así...y no come y le da que el jarabe y le da que esto y lo otro, le quita la ropa para que se refresque...uno no sabe como qué hacer...y al seguir así, a uno si le da tristeza" (C15).

El desespero también está presente en los datos “... y esa muchacha desesperada, desesperada, y es que yo no he podido enseñarle a ella, yo no he sido capaz... yo no sabía qué hacer..." (C2).

Las consecuencias de la angustia: el cuidado de un ser querido tiene repercusiones en la vida personal del cuidador. Las consecuencias se refieren a sentir culpa, perder el control y salir corriendo para buscar ayuda, ya sea para una consulta médica o para el hospital, cuando el cuidador no puede controlar la fiebre. El siguiente testimonio habla del sentimiento de culpa y sus motivos:

“...y cuando ya lo vieron así en mi casa...decían: "llevenlo a urgencias"...y mi hermano sí que es cierto...imagínese que era tanta la preocupación que él era regañando a mi mamá... y le echaba la culpa a ella...” ¡No! ¡Eso es culpa suya! porque sacándolo todos los días y eso asoliándolo...mi mamá me ayuda ...porque uno siendo uno primeriza y ella ya sabiendo más...Pues uno la deja hacer lo que ella sabe...pero muchas veces hay ocasiones que uno puede que sepa mas que ella...porque ella también se equivoca mucho..." (C 9).

En estas situaciones es razonable la pérdida del control:

...es la angustia (de) que lleva varios días con un antibiótico y entonces... ¿qué pasó? ¿Qué está pasando? ¿Por qué? ...por Dios ¿qué es? (C4).

La pérdida de control se contiene hasta que los cuidadores ya tienen una especie de agotamiento de sus recursos técnicos para cuidar al niño:

“...Al ver que pasen las horas y el niño sigue así...y no come y le da uno el jarabe y le da esto y lo otro, le quita la ropa para que se refresque...uno no sabe como qué hacer...y al seguir así... a uno si le da tristeza, que el niño no quiere comer, de verlo así de maluquito ...empieza como la tristeza de uno...ay Dios quiera que el niño se alivie, que se le quite la fiebre, que será lo que tiene... hay que pedirle una cita"(C15).

El punto crucial de la angustia: en la angustia hay un detonante o una especie de punto de quiebre el cual hace que se cambien las conductas, dando origen a la pérdida del control; esto sucede cuando el paciente tiene los $39^{\circ} \mathrm{C}$ de temperatura, es decir, que la fiebre llega a ese punto. Actitud del cuidador que se percibe tanto en el testimonio anterior como en el siguiente.

“Depende... que no sea $39^{\circ} \mathrm{C}, 39,5^{\circ} \mathrm{C}, 39,8^{\circ} \mathrm{C}$ ahí sí le da a uno susto... por convulsionar... no es que llegue allá y convulsione, es que de pronto... de pronto... Con $38^{\circ} \mathrm{C}, 38,5^{\circ} \mathrm{C}$, sí genera pues un poquito de angustia, pero no tanto que llegue a $39^{\circ} \mathrm{C}, 39,5^{\circ} \mathrm{C}$... ahí sí da una angustia que es más grande, porque de pronto puede convulsionar... "(C4).

Ante la situación descrita anteriormente, se puede salir corriendo a buscar ayuda, ya sea para la consulta o para el hospital como consecuencia de la angustia.

“... no tenés que apresurarte tanto... en este instante no tenés que salir corriendo con él... si... no te matés pa' salir corriendo con él, pero anda arreglándolo y te vas para el hospital..." (C6). 
Actitud que también se observa en las siguientes expresiones de los participantes:

“...me tocó consultar acá en el hospital, le mandaron [medicamento] venoso... estuve como tres días con él aquí... (C4). Yo soy más relajada y por eso me regañan... yo no consulto porque sí... yo consulto cuando no puedo hacer nada..." (C4).

La angustia del otro: en primer lugar, quién es ese otro. La angustia parece transmitirse mutuamente entre diferentes cuidadores, por una especie de empatía o resonancia: "pues no es lo mismo..., pero sí genera angustia... a mí sí me genera angustia... a mi si me genera angustia porque está mi mamá detrás del niño...angustiada y...ella también se angustia mucho y uno también se alcanza a angustiar..."(C 8).

\section{Fase analítica}

En esta fase se analizaron y compararon los datos sobre la angustia en el cuidador recogidos en la fase teórica, con los resultados obtenidos de las entrevistas con los cuidadores informales en el trabajo de campo. Seguidamente, se identificaron las características comunes que describen los antecedentes, atributos y consecuencias de la angustia en el cuidador informal las cuales se muestran en la tabla 4. Posterior a ello se describe la definición de la angustia en el cuidador. Es importante destacar que se encontraron ciertas características de los cuidadores que, si bien no son objeto del desarrollo del concepto, permiten su contextualización, son estas: la madre como figura paradigmática del cuidador informal y la corresidencia.

Por consiguiente, la angustia en el cuidador informal se define como un estado afectivo transitorio que surge en el cuidador que vive una condición de incertidumbre sobre algo desconocido que subyace al proceso febril, a las manifestaciones, al acompañamiento y a las consecuencias del signo o síntoma de la fiebre, provocando una sensación de zozobra (Ver tabla 4).

Tabla 4. Antecedentes, atributos y las consecuencias del concepto de angustia, identificados en el cuidador informal

\begin{tabular}{ll}
\hline & $\begin{array}{l}\text { Antecedentes } \\
\text { Incertidumbre, carga del cuidado, falta de apoyo, deterioro funcional de la persona que se cuida: ver el } \\
\text { niño diferente: "estar decaído, agitado, no querer comer y estar lloroncito", percepción de peligro. }\end{array}$ \\
$\begin{array}{c}\text { Concepto de } \\
\text { angustia en } \\
\text { el cuidador } \\
\text { informal }\end{array}$ & $\begin{array}{l}\text { Atributos } \\
\text { Impotencia, parálisis, confusión, tristeza, intranquilidad, zozobra, susto, miedo, ansiedad, preocupación, } \\
\text { estrés. } \\
\end{array}$ \\
& $\begin{array}{l}\text { Consecuencias } \\
\text { Sentir culpa, perder el control, angustia del otro, fatiga, trastornos del sueño, insomnio, vigilia, disminución } \\
\text { de la percepción de autoeficacia y autoestima, salir corriendo a buscar ayuda. }\end{array}$ \\
\hline
\end{tabular}

Fuente: Autor.

\section{Discusión}

Un aporte del análisis del concepto, se relaciona con la visibilización de la angustia en los cuidadores informales de niños con procesos agudos de enfermedad y en este caso, con la carga emocional que conlleva la experiencia de cuidado al niño con fiebre. Como se ilustra en los resultados, hay una coincidencia en las características definitorias de la angustia del cuidador informal de niños con enfermedades agudas y crónicas. El hallazgo de la angustia en los cuidadores de niños con fiebre se convierte en un reto para la enfermería, puesto que los cuidadores de niños en estados agudos de enfermedad como la fiebre, son también sujetos de cuidado que requieren la inclusión y el abordaje de acciones por parte de la enfermería que contribuyan a disminuir la angustia y la carga del cuidado que en ellos se presenta.

Una característica común encontrada en los cuidadores informales, es que son madres y conviven con el sujeto de cuidado. Sumado a esto, otras investigaciones plantean que la permanencia de forma continua con la persona que se cuida aumenta la percepción de sobrecarga en el cuidador, lo que puede dar origen a la presencia de la depresión y el deterioro de la salud. 
ISSN-PRINT

1794-9831

E-ISSN 2322-7028

Vol. 15 No. 2

Jul - Dic 2018

Cúcuta, Colombia
Diversos estudios muestran cómo la sobrecarga del cuidado $(7,8,16)$ surge como consecuencia de asumir la totalidad del cuidado de los hijos, además de las responsabilidades del hogar y del trabajo, lo cual disminuye las posibilidades de descanso e implica que el cuidador realice ajustes en su vida personal, ocasionándole serias complicaciones personales y para la familia, debido fundamentalmente a la responsabilidad implícita de cuidar del hijo enfermo.

Por otro lado se debe tener en cuenta, que si además de la angustia en el cuidador, el niño presenta problemas cognitivos, comportamentales, deterioro funcional, enfermedades graves y su condición de ser un niño, son factores a tener en cuenta exigiendo un incremento en los requerimientos del cuidador debido a que necesita un mayor acompañamiento y vigilancia (7).

La principal fuente de angustia es la incertidumbre sobre la enfermedad, que, para algunos autores, procede de su desconocimiento $(8,25)$. Para los cuidadores es de vital importancia saber qué está sucediendo, qué puede pasar y cómo puede resolver la situación en el momento. Este estudio indica que la incertidumbre se presenta por la inquietud sobre el desenlace y el impacto que pueda tener en el estado de salud del niño. Hallazgo que coincide con lo descrito en la teoría disciplinar de enfermería acerca de la incertidumbre ante la enfermedad de Mishel (32) la cual "tiene su apoyo más fuerte entre los sujetos que están experimentando la fase aguda de la enfermedad", caracterizada por la imposibilidad de identificar lo que sucede alrededor de la enfermedad y de predecir sus efectos. Esta situación es frecuente entre los cuidadores, la cual además se convierte en un desencadenante de la angustia que acompaña el proceso de cuidado, en casos relacionados con enfermedades infecciosas y en procesos vitales intensos como los accidentes y las crisis de diversa índole.

Este estudio coincide con otra investigación(5), que describe la presencia de la incertidumbre en todo el proceso de una enfermedad no diagnosticada, en la cual influyen también otros factores que contribuyen a empeorar la situación. Su análisis concuerda con el estudio, al determinar que los padres que perciben más incertidumbre, también experimentan menos control sobre la enfermedad de su hijo, e ilustran cómo su desconocimiento sobre la misma, así como el manejo contribuye a una incertidumbre significativa en toda la evolución de la fiebre en los niños.

De modo similar, también la incertidumbre produce susto y miedo (25), actitudes que se presentan ante algo desconocido que está agrediendo al niño, además de la tristeza y la ansiedad por la limitación para eliminar aquello que lo está afectando. De ahí que, se perciba la fiebre como una amenaza $(33,34)$ debido a los cambios observados en el niño que impresionan al cuidador y que por tanto crean un fuerte impulso por protegerlo del daño. El sentido de control de los padres y la amenaza que representa la fiebre (35) son factores que influyen sustancialmente en la forma de afrontar el cuidado. Se debe agregar además, que el miedo también se describe en diferentes estudios, como producto de los significados culturales que los cuidadores tienen sobre la fiebre y su relación con el daño cerebral y la muerte (36).

Hay que mencionar también, que los factores mencionados anteriormente pueden ocasionar en el cuidador impotencia que no lo deja actuar; igualmente, siente que desconoce lo que debe y puede hacer y que todo lo que ha realizado ha sido en vano al ver que el niño no mejora, lo que hace que se confunda y desespere con la subsecuente disminución de la percepción de autoeficacia y, a la vez, de autoestima, debido a que no sabe y no puede cuidar al paciente como él quisiera y debiera hacerlo (25).

Asimismo, la percepción permanente de amenaza se agrava cuando las acciones de cuidado y las subsiguientes secuelas son incontrolables, lo que genera impotencia (16) y desespero al no saber qué hacer de ahí en adelante; en consecuencia, la vigilia es permanente aumentando la carga física y emocional debido a que el cuidador está pendiente de tomar la temperatura al niño durante toda la noche. Hay que tener en cuenta, que el insomnio (21) es el resultado de la preocupación cuando hay un proceso febril en el niño, también es parte de la carga que se percibe con ciertos niveles de intensidad; igualmente, se manifiesta como una forma de estar atento, controlar los cambios en el niño y establecer acciones para protegerlo y confortarlo. De esta manera cuando el malestar o la enfermedad continúan, la preocupación y la tristeza (26) embargan al cuidador debido a que no puede lograr su objetivo de restablecer el equilibrio. 
Por otro lado, para los cuidadores el conocimiento de las complicaciones de la fiebre y la posibilidad de muerte del niño, se constituyen en uno de los principales factores de estrés y de angustia. La pérdida del control se evidencia cuando los cuidadores se enfrentan al fracaso respecto a los cuidados ofrecidos, lo que produce en ellos sentimientos de impotencia sobre el manejo del proceso febril, ocasionándoles sentimientos de culpa que se generan debido a la brecha existente entre el deseo de proteger a los niños y la realidad que se presenta (36).

También, se encuentra en otros estudios que cuando el control no se logra, se produce en el cuidador una situación de intranquilidad y desespero que no la puede manejar, decide entonces salir en busca de la ayuda de otro, llámese familiar o cuidador informal, formal, terapeuta o institución (37). Buscar ayuda es la consecuencia de haber perdido la capacidad de resolver la situación y no poder manejar la fiebre, sobretodo cuando se observa que los cuidados aplicados al niño no produjeron el efecto esperado. Es en este contexto donde los cuidadores consideran que el niño está en riesgo de convulsionar o morir, razón por la cual sienten la necesidad de traspasar el dominio sobre el proceso febril a otros que consideran poseen mayor experticia, entregando la responsabilidad del cuidado.

Habría que decir también que existe un sentimiento de desesperanza (3) el cual se puede transformar en otro como el de la culpa (4), estados de ánimo que afloran cuando el cuidado no da los resultados esperados; en este sentido, es tal la responsabilidad que está implícita en ese cuidado que todo lo que lo altere de manera negativa repercute en la búsqueda de un culpable, permitiendo que el cuidador pierda el control sobre el signo o síntoma y sobre la enfermedad.

De igual manera, se puede agregar que la angustia encontrada en los cuidadores, coincide con la "angustia realista" conceptualizada por Freud (2) como: "la defensa, el ataque, la parálisis y la huida son la respuesta a la angustia". En el estudio, las acciones que realizan los cuidadores, tienen como meta defender al niño de una posible amenaza, combatiendo la fiebre con las prácticas de cuidado que conocen; cuando no funcionan, la impotencia los invade al ver que pierden el control del proceso febril. Es por eso que el buscar ayuda se convierte en una forma de huir y de compartir la responsabilidad del cuidado del niño con otro, que lo considera lo suficientemente preparado, capaz de manejar mejor la situación de cuidado.

Para finalizar, vale la pena destacar que la angustia del cuidador en un proceso agudo, aunque sea una situación transitoria y probablemente no alcance de manera inmediata a repercutir en afecciones psicológicas ni físicas, como sí sucede con los cuidadores de niños con enfermedades crónicas que brindan de manera prolongada el cuidado, es relevante para la disciplina de enfermería la inclusión como se muestra en el estudio, debido a que es un fenómeno que tiene efectos en: el cuidador, en el niño y en los cuidados que se le brinda.

\section{Conclusiones}

- El análisis del concepto de angustia permitió aclarar su definición; establecer las semejanzas y diferencias con otros conceptos y la identificación de las circunstancias $\mathrm{y} / \mathrm{o}$ sentimientos que la anteceden, además de los atributos y las consecuencias que se derivan de la angustia del cuidador informal.

- Asimismo, se reconocieron las situaciones que pueden afectar la salud física y mental de los cuidadores cuando la angustia se prolonga, lo que permite a la disciplina de la enfermería generar aportes a la práctica de cuidado, especialmente en lo relacionado con la incertidumbre frente a la enfermedad.

- En la práctica, la angustia de los cuidadores tiene una existencia real, no se trata de invenciones ni de la imaginación del cuidador, esta angustia no es irracional. En ese sentido, desentrañar cómo ésta puede impactar la salud de los cuidadores, facilita diseñar a futuro estrategias de intervención que partan de la teoría, la investigación y la práctica.

- Finalmente, se debe tener en cuenta la angustia propia, la del otro, así como sus antecedentes y consecuencias, lo que significa que se debe incluir y reconocer al otro como sujeto de cuidado.

- En la práctica la aplicabilidad y estudio del concepto de angustia en los cuidadores informales, 
ISSN-PRINT

1794-9831

E-ISSN 2322-7028

Vol. 15 No. 2

Jul - Dic 2018

Cúcuta, Colombia es fundamental en los procesos nosológicos agudos y crónicos, y en todas aquellas situaciones de cuidado de enfermería y otras disciplinas que estén involucradas.

\section{Conflicto de Intereses}

Los autores declaran no tener ningún conflicto de intereses.

\section{Referencias Bibliograficas}

1. Ferrater Mora J. Dicionario de Filosofia. Buenos Aires, Argentina: Montecasino; 2014.

2. Freud S. Obras completas. Comentarios y notas de James Strachey con la colaboración de Anna Freud. Conferencias de introducción al psicoanálisis (Parte III) - (1916-1917) Volumen XVI. Paraguay: Amorrortu; 1976.

3. Velázquez Pérez Y, Espín Andrade AM. Repercusión psicosocial y carga en el cuidador informal de personas con insuficiencia renal crónica terminal. Rev Cuba salud pública [Internet]. 2014 [Consultado el 6 Julio de 2015]; 40(1):3-17. Disponible en: http://scielo.sld.cu/scielo.php?script=sci arttext\&pid $=\mathrm{S} 0864-34662014000100002$

4. Ávila Toscano JH, Vergara Mercado M. Calidad de vida en cuidadores informales de personas con enfermedades crónicas. Aquichan [Internet]. 2014 [Consultado el 8 de Junio de 2016]; 14(3):417-429. Disponible en: http: http://aquichan.unisabana.edu.co/index.php/aquichan/article/view/2967

5. Malpert AV, Kimberg C, Luxton J, Mullins LL, Pui CH, Hudson MM, et al. Emotional distress in parents of long-term survivors of childhood acute lymphoblastic leukemia. Psychooncology. 2015; 24(9):1116-23.

6. Zarit SH, Reever KE, Bach Peterson J. Relatives of the Impaired Elderly: Correlates of Feelings of Burden. Gerontologist [Internet]. 1980 [cited 2016 Oct 3]; 20(6):649-655. Available from: https:// academic.oup.com/gerontologist/article-abstract/20/6/649/629680

7. Kelley SJ, Whitley DM, Campos PE. Psychological Distress in African American Grandmothers Raising Grandchildren: The Contribution of Child Behavior Problems, Physical Health, and Family Resources. Res Nurs Heal. 2013; 36(4):373-385.

8. Pedrón Giner C, Calderón C, Martínez Costa C, Borraz Gracia S, Gómez López L. Factors predicting distress among parents/caregivers of children with neurological disease and home enteral nutrition. Child Care Heal Dev. 2014; 40(3):389-397.

9. Macedo EC, Rangel da Silva L, Santos Paiva M, Pereira Ramos MN. Burden and quality of life of mothers of children and adolescents with chronic illnesses: an integrative review. Rev Latino-Am Enferm [Internet]. 2015 [cited 2016 Jun 16]; 23(4):769-777. Available from: http://www.scielo.br/ scielo.php?script $=$ sci arttext\&pid=S0104-11692015000400769\&lng=en\&tlng=en

10. Molzon ES, Mullins LL, Cushing CC, Chaney JM, McNall R, Mayes S. The relationship between barriers to care, caregiver distress, and child health-related quality of life in caregivers of children with cancer: A structural equation modeling approach. Child Heal Care [Internet]. 2016 [cited 2016 Jun 16]; 0(0):1-15. Available from: http://dx.doi.org/10.1080/02739615.2016.1275639

11. Cantón Hernández JC. Características y necesidades de las personas en situación de dependencia. Madrid, España: Editex; 2014.

12. Zarit SH, Reever KE, Bach Peterson J. Relatives of the Impaired Elderly: Correlates of Feelings of Burden. Gerontologist [Internet]. 1980 [cited 2016 Oct 3]; 20(6):649-55. Available from: https:// academic.oup.com/gerontologist/article-abstract/20/6/649/629680

13. Schwartz Barcott D, Kim H. An expansion and elaboration of the hybrid model of concept development. In: Concept Development in nursing Foundations, Techniques and Applications. 2a ed. W.B. Saunders Company; 2000. 
14. Oxford Advanced Learner's Dictionary anguish [Internet] 2017. [cited 2017 Jul 9]. Available from: http://www.oxfordlearnersdictionaries.com/definition/english/anguish?q=anguish

15. Real Academia Española. Diccionario de la Lengua Española. 23 ed [Internet]. 2014 [Consultado el 2 Febrero de 2015]. Disponible en: http://dle.rae.es/?id=2fiyliB

16. Nam GE, Warner EL, Morreall DK, KirchhoffAC, Kinney AY, Fluchel M. Understanding psychological distress among pediatric cancer caregivers. Support Care Cancer. 2016; 24(7):3147-55.

17. Ayuso Gutiérrez JL. Trastornos de angustia. Barcelona, España: Martínez Roca; 1988.

18. Jaser SS, Linsky R, Grey M. Coping and psychological distress in mothers of adolescents with type 1 diabetes. Matern Child Heal J. 2014;18(1):101-8.

19. Barrera Ortiz L, Sánchez Herrera B, Carrillo González GM. La carga del cuidado en cuidadores de niños con enfermedad crónica. Rev Cuba enferm [Internet]. 2013 [Consultado el 15 Mayo de 2017]; 29(1):39-47. Disponible en: http://scielo.sld.cu/scielo.php?script=sci_arttext\&pid $=\mathrm{S} 0864-03192013000100006$

20. Olagunju AT, Asoegwu CN, Campbell EA, Akinbode AA, Aina OF, Nwawolo CC. Impact of emotional distress on caregivers burden among Nigerian children with Obstructive Adenotonsillar hypertrophy. Int J Pediatr Otorhinolaryngol. 2015; 79(6):858-62.

21. Vieira Figueiredo S, Carvalho de Sousa AC, Verde Gomes IL. Children with special health needs and family: implications for Nursing. Rev Bras Enferm [Internet]. 2016 [cited 2017 Feb 8]; 69(1):79-85. Available from: http://dx.doi.org/10.1590/0034-7167.2016690112i

22. Choi EK, Yoon SJ, Kim JH, Park HJ, Kim JY, Yu ES. Depression and distress in caregivers of children with brain tumors undergoing treatment: Psychosocial factors as moderators. Psychooncology. 2016; 25(5):544-50.

23. Ma GY, Mak WW. Caregiving-specific worry, affiliate stigma, and perceived social support on psychological distress of caregivers of children with physical disability in Hong Kong. Am J Orthopsychiatry. 2016; 86(4):436-446.

24. Al Gamal E, Long T. Psychological distress and perceived support among Jordanian parents living with a child with cerebral palsy: A cross-sectional study. Scand J Caring Sci [Internet]. 2013 [cited 2015 Feb 18]; 27(3):624-631. Available from: https://www.ncbi.nlm.nih.gov/pubmed/22924549

25. Ross A, Yang L, Klagholz SD, Wehrlen L, Bevans MF. The relationship of health behaviors with sleep and fatigue in transplant caregivers. Psychooncology. 2016; 25(5):506-12.

26. Michelson N, Riis JL, Johnson SB. Subjective Social Status and Psychological Distress in Mothers of Young Children. Matern Child Health J. 2016; 20(10):2019-2029.

27. Teixeira RJ, Pereira MG. Psychological morbidity and autonomic reactivity to emotional stimulus in parental cancer: A study with adult children caregivers. Eur J Cancer Care (Engl). 2014; 23(1):129139.

28. Mosher CE, Given BA, Ostroff JS. Barriers to mental health service use among distressed family caregivers of lung cancer patients. Eur J Cancer Care (Engl). 2015; 24:50-59.

29. Runyon MK, Spandorfer ED, Schroeder CM. Cognitions and distress in caregivers after their child's sexual abuse disclosure. J Child Sex Abus. 2014; 23(2):146-159.

30. Doulavince Amador D, Pimentel Gomes I, Pereira da Silva Reichert A, Collet N. Repercussões do câncer infantil para o cuidador familiar: revisão integrativa. Rev Bras Enferm [Internet]. 2013 [cited 2016 May 5]; 66(2):267-270. Available from: http://www.scielo.br/scielo.php?script=sci arttext\&pid $=$ S0034-71672013000200017\&lng=en\&nrm $=$ iso\&tlng $=p t$

31. Kerns CE, Elkins RM, Carpenter AL, Chou T, Green JG, Comer JS. Caregiver distress, shared traumatic exposure, and child adjustment among area youth following the 2013 Boston Marathon bombing. J Affect Disord. 2014; 167:50-55. 
32. Mishel MH. Reconceptualization of the Uncertainty in Illness Theory. Image J Nurs Sch. 1990; 22(4):256-262.

33. Langer T, Pfeifer M, Soenmez A, Kalitzkus V, Wilm S, Schnepp W. Activation of the maternal caregiving system by childhood fever-a qualitative study of the experiences made by mothers with a German or a in the care of their children. BMC fam Pr [Internet]. 2013 [cited 2015 Feb 26]; 14:35. Available from: https://www.ncbi.nlm.nih.gov/pmc/articles/PMC3607993/

34. Teagle AR, Powell CVE. Is fever phobia driving inappropriate use of antipyretics? Arch Dis Child. 2014; 99(7):701-702.

35. Sahm LJ, Kelly M, McCarthy S, O’Sullivan R, Shiely F, Romsing J. Knowledge, attitudes and beliefs of parents regarding fever in children: a Danish interview study. Acta Paediatr. 2016; 105(1):69-73.

36. Arruda Colli MNF, Perina EM, Santos MA. Experiences of Brazilian children and family caregivers facing the recurrence of cancer. Eur J Oncol Nurs. 2015; 19(5):458-464.

37. Cinar ND, Altun I, Altınkaynak S, Walsh A. Turkish parents' management of childhood fever: A crosssectional survey using the PFMS-TR. Australas Emerg Nurs J [Internet]. 2014 [cited 2015 Jun 9]; 17(1):3-10. Available from: http://www.aenj.com.au/article/S1574-6267(13)00104-3/pdf 